\title{
Non-isotropic distance measures for lattice-generated sets
}

\author{
A. Iosevich and M. Rudnev
}

December 23, 2004

\begin{abstract}
We study distance measures for lattice-generated sets in $\mathbb{R}^{d}, d \geq 3$, with respect to nonisotropic distances $|\cdot|_{K}$, induced by smooth symmetric convex bodies $K$. An effective Fourieranalytic approach is developed to get sharp upper bounds for the second moment of the weighted distance measure.

The implications of these estimates are discussed in the context of the general Erdös-Falconer distance problem.
\end{abstract}

Keywords Lattice point distribution, mean square estimates, distance measures, homogeneous sets AMS subject classification $42 \mathrm{~B}$

\section{Introduction}

1.1 In this paper we study distance sets, corresponding to the integer lattice $\mathbb{Z}^{d}, d \geq 3$, with respect to non-isotropic distances, defined in terms of the Minkowski functional of some well-curved central-symmetric convex body $K \subset \mathbb{R}^{d}$, with a smooth boundary $\partial K$. Namely we assume that the Gaussian curvature on the boundary $\partial K$ is bounded from above and below by some fixed pair of positive constants. The boundary itself should be $C^{r}$, for a large enough $r$, and we do not discuss how small $r$ can possibly be. Suppose the volume of $K$ equals the volume of the Euclidean unit ball, which is denoted as $B$ throughout the paper. Let $\mathcal{K}$ denote the class of such convex bodies.

For $K \in \mathcal{K}$, let $|\cdot|_{K}$ be the Minkowski functional of $K$, or the $K$-norm. Let $|\cdot|_{K^{*}}$ be the dual norm to $|\cdot|_{K}$, defined as

$$
|x|_{K^{*}}=\sup _{y \in K}|x \cdot y|, \quad K_{*}=\left\{x \in \mathbb{R}^{d}:|x|_{K^{*}} \leq 1\right\} .
$$

The dual $K^{*}$ also belongs to the class $\mathcal{K}$.

For a Borel set $S \subset \mathbb{R}^{d}$, define its $K$-distance set as

$$
\Delta_{K}(S)=\left\{|a-b|_{K}: a, b \in S\right\} .
$$

Let $\Delta(S) \equiv \Delta_{B}(S)$ be the distance set of $S$ with respect to the Euclidean metric $\|\cdot\|$.

An infinite discrete set $A \subset \mathbb{R}^{d}$ is called homogeneous if all its elements are separated by some $c>0$, while any cube of side length $C>c$ contains at least one element of $A$.

Let $q \gg 1$ be a large real, consider a homothety $q K$ of $K$, with respect to the center of $K$. For a homogeneous discrete set $A$, let $A_{q}=A \cap q K$ be a truncation of $A$ (which strictly speaking depends on $K)$. 
In the special case of $A=\mathbb{Z}^{d}$ and $K=B$, it is well known that

$$
\# \Delta\left(A_{q}\right) \approx \begin{cases}q^{2}, & d \geq 3, \\ \frac{q^{2}}{\sqrt{\log q}}, & d=2 .\end{cases}
$$

To fix the notation, \# denotes the cardinality of a finite set, $|\cdot|$ stands for the Lebesgue measure of a Borel measurable set. The symbols $O$ or $\lesssim$ absorb constants depending only on $K$ (and hence $d$ ). Also we write $a \gtrsim b$, or equivalently $a=\Omega(b)$, if $b \lesssim a$ and $a \approx b$ if both $a \lesssim b$ and $a \gtrsim b$. The symbol $\sim$ will indicate proportionality, up to some constant $c(K)$.

The main goal in this paper is to develop a technically transparent Fourier-analysis based approach which would extend estimate (3) to the case of $K$-distances. We are able to do so in $d \geq 4$; if $d=3$ we are off by a logarithmic factor. The result follows by estimating the second moment of the corresponding distance measure; it is stated in Theorem 2 further in the paper, after all the notations have been developed.

Theorem 1 If $A=\mathbb{Z}^{d}$, the d-dimensional integer lattice, one has

$$
\# \Delta_{K}\left(A_{q}\right) \gtrsim \begin{cases}q^{2}, & d \geq 4 \\ q^{2} \log ^{-2} q, & d=3\end{cases}
$$

1.2 Theorem 1 can be given interpretation in terms of the borderline dimension $\frac{d}{2}$ in the Falconer distance problem. The Falconer distance problem states that if the Hausdorff dimension of a Borel set $S \subset \mathbb{R}^{d}, d \geq 2$ is greater than $\frac{d}{2}$, then the Lebesgue measure of the distance set $|\Delta(S)|>0$. (See [5], [11], [2], [16], [17], [3], and the references contained therein for the description of this open for every $d \geq 2$ problem and progress over the years. The best known results are due to Wolff in $\mathbb{R}^{2}$ and Erdogan in $\mathbb{R}^{d}$, who vindicate the conjecture if the dimension of $S$ exceeds $\frac{d(d+2)}{2(d+1)}$ rather than $\frac{d}{2}$.) The discrete analogue of the Falconer distance problem is the Erdös distance conjecture (see e.g. [13] and the references contained therein for thorough discussion and the state-of-the-art) restricted to homogeneous sets, which states

$$
\# \Delta\left(A_{q}\right) \geq C_{\varepsilon} q^{2-\varepsilon} .
$$

Falconer ([5]) showed that the borderline dimension $\frac{d}{2}$ cannot be improved, due to the following construction. Fix a rapidly growing sequence of positive integers $\left\{q_{i}\right\}_{i \geq 1}$, with $q_{1}=2$ and $q_{i+1}>q_{i}^{i}$. Let $A=\mathbb{Z}^{d}$ and $S_{i}$ be the union of Euclidean balls of radius $q_{i}^{-\frac{d}{s}}$, for some $0<s<d$, centered at the points of $\frac{1}{q_{i}} A_{q_{i}}$. Let us call $S_{F}=\cap_{i} S_{i}$ the Falconer set. Then (see e.g. [4]) the Hausdorff dimension $\operatorname{dim}_{\mathcal{H}} S_{F}=s$. On the other hand, the Lebesgue measure

$$
\left|\Delta\left(S_{i}\right)\right| \approx q_{i}^{-\frac{d}{s}} \cdot \# \Delta\left(A_{q_{i}}\right) .
$$

It follows from (3) that $\left|\Delta\left(S_{F}\right)\right|=0$ if $s<\frac{d}{2}$. More precisely, $\left|\Delta\left(S_{F}\right)\right|>0$, provided that $s \geq \frac{d}{2}$ for $d \geq 3$ and $s>\frac{d}{2}$ for $d=2$.

An immediate consequence of Theorem 1 is that the same conclusion can be drawn in the case $d \geq 4$ for the Falconer set $S_{F}$ with respect to $K$-distances, $K \in \mathcal{K}$, irrespective of the pair of constants that bound the curvature. Consequently, as the basis for the Falconer construction one can use any $d$-dimensional lattice, in which case Theorem 1 will be valid as well. 
Corollary 1.1 Let $S_{F}$ be the Falconer set with $\operatorname{dim}_{\mathcal{H}} S_{F}=s$. Suppose, $s \geq \frac{d}{2}$, and $d \geq 4$. Then $\left|\Delta_{K}\left(S_{F}\right)\right|>0$, for any central-symmetric $K$, which is smooth and has bounded and everywhere non-zero curvature on $\partial K$.

Remark: The assumption of central symmetry is not essential: one can always consider $\mathbb{Z}_{+}^{d}$ only. We do not study to what extent the curvature assumption can be weakened so that $K$ may still qualify as "well-curved", which for our purposes is determined by the validity of Lemma 2.2 further in the paper.

As a separate issue, our result shows that if one fixes the Euclidean metric $\|\cdot\|$, the scope of Falconer's construction can be extended to a class $\mathcal{A}$ of homogeneous sets

$$
A=\left\{\frac{|a|_{K}}{\|a\|} a: a \in \mathbb{Z}^{d}\right\}, K \in \mathcal{K} .
$$

The difficult part in Theorem 1 is the endpoint issue, namely the precision of the exponents in estimates (4), which transcribes into the inequality $s \geq \frac{d}{2}$, inclusive of the endpoint, in Corollary 1.1. Otherwise, that is if an extra $q^{\varepsilon}$ were allowed in the right-hand side of (4), the proof can be made somewhat shorter, using the techniques developed by Müller ([12]) and Iosevich et al. ([8]) developed to study the quantity

$$
\mathcal{E}(t)=\#\left\{t K \cap \mathbb{Z}^{d}\right\}-t^{d} \operatorname{Vol} K
$$

1.3 It appears to be important to understand how much the analytic methods for geometry of numbers, i.e. in the present context the case $A=\mathbb{Z}^{d}$, can apply to study the Erdös-Falconer problem, dealing with general homogeneous sets $A$. The motivation for doing so it comes from a hypothesis closely related to (5) that lattice sets yield local minima for second moments of the corresponding distance measures, with respect to variations of the sets. Hence our approach is developed on the basis of the general distance measure formalism, set up by Mattila ([11]). The distance measure $\nu(t)$, relative to the set $A_{q}$ counts the number of points of $A_{q}$ in $\frac{1}{q}$-thin $K$-annuli of radius $t$, centered at points of $A_{q}$, average with respect to the position of the center. (In the case of a lattice it suffices to fix the center at the origin). The $L^{1}$-norm $\|\nu\|_{1}$ is approximately the number of points of $A$ in $q K$. The main task is to estimate the square of $L^{2}$-norm, or the second moment $\|\nu\|_{2}^{2}$. This is the content of the forthcoming Theorem 2, after all the definitions have been made. Beyond this non-technical introduction, we will be using various weighted measures $\nu$, which will carry extra identification.

The distance measure formalism however has nothing to do with the lattice structure, in the sense that any finite compactly supported Borel measure $\mu$ in $\mathbb{R}^{d}$ generates a well-defined distance measure $\nu$ in $\mathbb{R}_{+}$. To this effect, Mattila ([11]) proved a general theorem for the Euclidean distance, which generalizes to $K$-distances (see [1]) as follows. The second moment of the $K$-distance measure $\nu$, generated by $\mu$ is finite in case what we call the Mattila integral

$$
M(\mu)=\int_{1}^{\infty}\left(\int|\hat{\mu}(t x)|^{2} d \omega_{K^{*}}(x)\right)^{2} t^{d-1} d t<\infty .
$$

Above, $\omega_{K^{*}}$ is the Lebesgue measure on $\partial K^{*}$. Hence, if $M(\mu)<\infty$, the Lebesgue measure of the support of $\nu$ is positive. As for the Falconer construction (see 1.2), for the natural (i.e. induced by the Lebesgue measure in $\mathbb{R}^{d}$ ) measure on the set $S_{F}$, in the case $\operatorname{dim}_{\mathcal{H}} S_{F}<\frac{d}{2}$ the integral (9) diverges. 
In essence, our proof of Theorem 2 consists in analyzing and estimating the Mattila integral for a natural measure $\mu$ on the set $S_{i}=S_{q_{i}}$, in the Falconer construction, when $S_{F}=\cap S_{i}$ has Hausdorff dimension $s=\frac{d}{2}$, after fixing $q_{i}=q$ and appropriate scaling.

Looking back at the construction of the set $S_{F}$, it is clear that instead of the lattice $\mathbb{Z}^{d}$, one can use any homogeneous set $A$ as a basis for the construction. Unfortunately, our proof of Theorem 2 does not extend beyond the very special case when $A$ is a lattice. The reason is that in the proof we use a smooth approximation $E(t)$ of the discrepancy $\mathcal{E}(t)$, see $(8)$, as the auxiliary quantity. In the lattice case, $E(t)$ admits a well known analytic representation via the Poisson summation formula, whereupon $E^{2}(t)$ looks very similar to the integrand in the Mattila integral. It follows that $L^{2}$ estimates for the distance measure $\nu$ can be obtained in terms of $L^{2}$-estimates for $E$. Whether such an approach has a prototype in the general homogeneous set context is not at all clear.

1.4 To prove our main result we develop an asymptotic method, which enables one first to dominate the $L^{2}$-estimate for $E$ by a weighted $L^{2}$ estimate for $\nu$, see (46) below. Then the latter estimate can in turn be dominated by another $L^{2}$-estimate for $E$, see e.g. (62) below. Theorem 2 follows. $L^{2}$-estimates for the quantity $E$ were obtained in the works [12] and [8] (see also [9] for further developments) where basically the same trick was used. However, the asymptotic techniques of those papers did not yield a clear cut relation like (46) between the $L^{2}$ estimates for the quantities $\nu$ and $E$, due to plethora of cut-off functions, truncations, etc. used. These are the technical difficulties one encounters in the effort to attain the endpoint result claimed in Theorem 1 . We identify the estimate (46) as the key display of the technical advantage of our approach, which also yields the mean square estimates for $E$ and $\mathcal{E}$ as a by-product. In addition, throughout the proof a number of integral representations for the distance measure $\nu$ and related quantities are obtained, which can be interesting in their own right. The approach rests on the use of the Hankel rather than Fourier transform for distance measures, defined on $\mathbb{R}_{+}$, which enables to make the analysis fairly transparent.

Due to the fact that it is only a weighted estimate for the second moment of the distance measure $\nu$ that gets majorated by the second moment of $E$, our approach results in tight (modulo the logarithmic factor in $d=3$ ) estimates for the second moment of $\nu$ in $d \geq 3$, yet for $d=2$ it does not do better than yield a trivial estimate. In $d=2$, the case of a general $K$ is an open problem.

The main body of the paper is organized as follows. In Section 2 we set up the distance measure formalism in the context of a general homogeneous set $A$. In the special case $A=\mathbb{Z}^{d}$, we formulate Theorem 2 and show how it implies Theorem 1. As an example of how the formalism applies to a general $A$, we briefly discuss the Euclidean distance case and write out the integral expression for the second moment of the distance measure. We further move on to the case of $K$-distances and prove the general Mattila integral identity for the second moment in Proposition 2.3. The proposition contributes little to the special case $A=\mathbb{Z}^{d}$. However, it establishes the proof template which is further used in Section 3 to prove the crucial Lemma 3.1. Section 3 however is already fully dedicated to the case $A=\mathbb{Z}^{d}$ and from its outset takes advantage of the Poisson summation formula. Comparison of the yield of the Poisson summation formula for the distance measure with the general formula in Proposition 2.3 yields as a by-product Theorem 3 on duality. However, the main result of Section 3 is Lemma 3.1. In Section 4 this lemma is used to prove Theorem 2.

\section{Distance measure}

Let $\phi$ be a non-negative radial (radial henceforth means radial with respect to the Euclidean metric) Schwartz class function, such that $\int \phi(x)=1, \phi(x)=1$ inside the ball of some radius and vanishes 
outside the ball of twice the radius. Let $q$ be a large number, denote $\phi_{q}(x)=q^{d} \phi(q x)$ and $A \subset \mathbb{R}^{d}$ a homogeneous set, $A_{q}=A \cap q K$. Let also $\mathbb{Z}_{q}^{d}=\mathbb{Z}^{d} \cap q K$ for the special case $A=\mathbb{Z}^{d}$. Without loss of generality (only to discount additional trivial estimates) suppose $A_{q}$ contains no point in some $c$-neighborhood of the origin.

For a function $f \in L^{1}\left(\mathbb{R}^{d}\right) \cap L^{2}\left(\mathbb{R}^{d}\right)$, let

$$
\hat{f}(\xi)=\int f(x) e^{-2 \pi \iota \xi \cdot x} d x
$$

define the Fourier transform. Let

$$
\mu_{q}(x)=\sum_{a \in A_{q}} \phi_{q}(x-a)
$$

be the smoothing of the counting measure on $A_{q}$. The radius of the atoms of the measure $\mu_{q}$ is $c_{0} / q$, with $0<c_{0}<1$ by the choice of $\phi$. Clearly

$$
\hat{\mu}_{q}(\xi)=\sum_{a \in A_{q}} \hat{\phi}(\xi / q) e^{-2 \pi \iota a \cdot \xi}
$$

and the function $\hat{\phi}$ is radial.

To study the distances between the elements of $A$ and the origin, define for $t>0$ :

$$
\begin{aligned}
\nu_{q, 0}(t) & =\int \omega_{K}(x / t) d \mu_{q}(x), \\
N_{q, 0}(t) & =\int \Omega_{K}(x / t) d \mu_{q}(x)=\int_{0}^{t} d \nu_{q, 0} .
\end{aligned}
$$

Above $\omega_{K}$ is the Lebesgue measure on $\partial K, \Omega_{K}$ is the characteristic function of $K$. Note that in the first integral $\mu_{q}$ is actually a Schwartz function, and $\omega_{K}$ - a distribution.

Without loss of generality one can assume that every lattice cube contains exactly one point of $A$ (this can always be achieved for any finite truncation $A_{q}$ by sparsing it out and subsequent scaling). In this case define the volume discrepancy

$$
E_{q, 0}(t)=N_{q, 0}(t)-t^{d} \operatorname{Vol} K
$$

Studying the quantity $E_{q, 0}$ for the integer lattice has a long history, see [8] for some references. In the general context of homogeneous sets, the quantity $E_{q, 0}$ defined relative to the origin cannot be expected to be smaller in absolute value than $O\left(q^{d-1}\right)$. However, averaging with respect to the choice of the center throughout $A_{q}$ can result in a non-trivial estimate, important in the context of the Erdös distance problem. This issue is briefly discussed further in the paper following (30).

The seemingly redundant 0 subscripts come from the fact that in the sequel it turns out to be more convenient to work with the weighted quantities

$$
\left[\nu_{q}(t), N_{q}(t), E_{q}(t)\right]=t^{\frac{1-d}{2}}\left[\nu_{q, 0}, N_{q, 0}(t), E_{q, 0}(t)\right]
$$

The quantity $\nu_{q, 0}$ is the density of the measure $\mu_{q}$ on $K$-spheres of radius $t$, centered at the origin. The primitive $N_{q, 0}(t)$ counts the points in $K$-balls of radius $t$. Clearly

$$
\int_{0}^{\infty} \nu_{q, 0} \sim q^{d} .
$$


By definition of the quantities $\mu_{q}$ and $\nu_{q, 0}$, in order to obtain estimates in terms of $q$, it is legitimate to sample integrals containing $\nu_{q, 0}$ (as well as $E_{q, 0}$ and other versions of $\nu$ and $E$ to appear later) by Darboux sums with the step size $\frac{1}{c_{1} q}$, for some constant $c_{1}$.

Clearly $\nu_{q, 0}$ vanishes for $t>q+q^{-1}$, while for $t<q-q^{-1}$,

$$
\nu_{q, 0} \approx q^{-1} \Gamma\left(t, q^{-1}\right),
$$

where $\Gamma(t, \delta)$ is the number of points of $A$ in a $K$-annulus $\alpha(t, \delta)$ (defined as $(t+\delta) K \backslash t K$ ) centered at the origin, with radius $t$ and width $\delta$; further on $\delta$ will always be approximately $\frac{1}{q}$. More precisely the statement (17) means that there exist uniform constants $c_{2}$ and $c_{3}$, such that

$$
\Gamma\left(t, \frac{1}{c_{2} q}\right) \leq \frac{\nu_{q, 0}}{q} \leq \Gamma\left(t, \frac{c_{3}}{q}\right)
$$

Let us cover $A_{q}$ by a set of concentric $K$-annuli $\alpha_{k}$ around the origin, all of which have fixed width $\delta \sim \frac{1}{q}$. Suppose $\alpha_{1}$ has radius $q^{-1}$ and for $k>1$ the inner boundary of $\alpha_{k+1}$ coincides with the outer boundary of $\alpha_{k}$. Terminate the construction as soon as $q K$ is covered by the union of $\alpha_{k}$. Thus $k \lesssim q^{2}$ and the $K$-radii $t_{k}$ of $\alpha_{k}$ go up to $q+O\left(q^{-1}\right)$. Define the annulus standard deviation and $D_{\alpha}$ and the ball mean square discrepancy $D_{K}$ as follows:

$$
\begin{aligned}
& D_{\alpha}=\sqrt{\frac{1}{q^{2}} \sum_{k} \Gamma^{2}\left(t_{k}, \delta\right)} \approx \sqrt{\frac{1}{q^{3}} \int_{0}^{q} \nu_{q, 0}^{2}(t) d t}, \\
& D_{K}=\sqrt{\frac{1}{q^{2}} \sum_{k} E_{q, 0}^{2}\left(t_{k}\right)} \approx \sqrt{\frac{1}{q} \int_{0}^{q} E_{q, 0}^{2}(t) d t} .
\end{aligned}
$$

Theorem 2 Suppose $A=\mathbb{Z}^{d}$. Then

$$
\begin{array}{ll}
D_{\alpha}, D_{K} \lesssim q^{d-2}, & d \geq 4, \\
D_{\alpha}, D_{K} \lesssim q \log q, & d=3 .
\end{array}
$$

As far as the weighted quantity $\nu_{q}(t)$ is concerned, see (15), the estimate (20) of Theorem 2 is tantamount to

$$
\left\|\nu_{q}\right\|_{2}^{2}=\int_{0}^{\infty} \nu_{q}^{2}(t) d t \lesssim \begin{cases}q^{d}, & d \geq 4 \\ q^{3} \log ^{2} q, & d=3 .\end{cases}
$$

Theorem 2 implies Theorem 1.

Proof of Theorem 1 Assume Theorem 2. By the Cauchy-Schwartz inequality,

$$
q^{2 d} \approx\left(\int_{1}^{q} \nu_{q, 0} d t\right)^{2} \leq\left|\operatorname{supp} \nu_{q, 0}\right| \int_{1}^{q} \nu_{q, 0}^{2}(t) d t
$$

where $\left|\operatorname{supp} \nu_{q, 0}\right|$ is the Lebesgue measure of the support of $\nu_{q, 0}$. Substituting the estimates (20) in the right hand side, one gets the lower bound $\left|\operatorname{supp} \nu_{q, 0}\right| \gtrsim q$ for $d \geq 4$ and $\left|\operatorname{supp} \nu_{q, 0}\right| \gtrsim \frac{q}{\log ^{2} q}$ for $d=3$. Hence, by definition of $\nu_{q, 0}$, cf. (17), there exists $\Omega\left(q^{2}\right)$ in $d \geq 4$ and $\Omega\left(q^{2} / \log ^{2} q\right)$ disjoint $K$-annuli, of width $\delta \sim \frac{1}{q}$, and whose radii do not exceed $q$, such that each of these annuli contains at least one lattice point. This is equivalent to the statement of Theorem 1. 
Let us now return to the general homogeneous set $A$ set-up. Observe that in the same way as (22), as $\left|\operatorname{supp} \nu_{q, 0}\right| \lesssim q$, for the second moment of the weighted quantity $\nu_{q}$ one should always have

$$
\left\|\nu_{q}\right\|_{2}^{2} \gtrsim q^{d}
$$

Let us write up some integral representations for the quantities $\nu_{q}, N_{q}$. Applying the Plancherel theorem to the integrals in (13), for the weighted quantities (15) we get:

$$
\begin{aligned}
& \nu_{q}(t)=t^{\frac{d-1}{2}} \int \hat{\phi}(\xi / q) \hat{\omega}_{K}(t \xi) \sum_{a \in A_{q}} e^{-2 \pi \iota a \cdot \xi} d \xi, \\
& N_{q}(t)=t^{\frac{d+1}{2}} \int \hat{\phi}(\xi / q) \hat{\Omega}_{K}(t \xi) \sum_{a \in A_{q}} e^{-2 \pi \iota a \cdot \xi} d \xi .
\end{aligned}
$$

Observe that $\nu_{q}(t)$ extends as zero to $t=0$, as well as the fact that the quantity $N_{q}(t)$ is not in $L^{2}\left(\mathbb{R}_{+}\right)$if $d=2$.

\section{Euclidean case}

First let us get an integral representation for the second moment $\left\|\nu_{q}\right\|_{2}^{2}$ when $K$ is the Euclidean ball, with the notations $\omega_{B}, \Omega_{B}$ for the surface and volume measure. In this case the Fourier transform $\hat{\omega}_{B}(\xi)$ is radial, namely $\hat{\omega}_{B}(\xi) \sim\|\xi\|^{1-\frac{d}{2}} J_{\frac{d}{2}-1}(2 \pi\|\xi\|)$, where $J_{v}$ further denotes the Bessel function of order $v \geq 0$. Let us skip the factor of $2 \pi$ in what follows. This can always be accomplished by scaling. After writing the integral (24) for $\nu_{q}$ in the spherical coordinates we have

$$
\nu_{q}(t) \sim \sqrt{t} \int_{0}^{\infty} r J_{\frac{d}{2}-1}(r t) \psi(r / q) \sum_{a \in A_{q}} J_{\frac{d}{2}-1}(r\|a\|) d r
$$

where henceforth

$$
\psi(r)=\left.\hat{\phi}(\xi)\right|_{\|\xi\|=r},
$$

so $|\psi(r / q)|$ is asymptotically smaller than any inverse power of $r / q$.

Using the Hankel formula (see e.g. [15]),

$$
\int_{0}^{\infty} t J_{v}(a t) J_{v}(b t) d t=\frac{\delta(a-b)}{a}
$$

one gets from $(24)$

$$
\begin{aligned}
\left\|\nu_{q}\right\|_{2}^{2}=\int_{0}^{\infty} \nu_{q}^{2}(t) d t & \sim \int_{0}^{\infty} r \psi^{2}(r / q) \sum_{a, b \in A_{q}} \frac{J_{\frac{d}{2}-1}(r\|a\|) J_{\frac{d}{2}-1}(r\|b\|)}{(\|a\|\|b\|)^{\frac{d}{2}-1}} d r \\
& \sim \int_{0}^{\infty} r^{d-1} \psi^{2}(r / q) \sum_{a, b \in A_{q}} \hat{\omega}_{B}(r\|a\|) \hat{\omega}_{B}(r\|b\|) d r .
\end{aligned}
$$

The representation (28) is closely related to the Mattila integral (9) for the measure $\mu_{q}$.

Observe that by the Hankel formula (27) with $v=\frac{d}{2}-1$, the expression (28) is in essence the Parseval identity for the Hankel transformation

$$
H\left[\nu_{q}\right](r)=\int_{0}^{\infty} \sqrt{r t} J_{\frac{d}{2}-1}(r t) \nu_{q}(t) d t \sim \sqrt{r} \psi(r / q) \sum_{a \in A_{q}} \frac{J_{\frac{d}{2}-1}(r\|a\|)}{(\|a\|)^{\frac{d}{2}-1}} .
$$


Remark Similarly to (28) one can apply the Hankel formula with $v=\frac{d}{2}$ to the quantity $N_{q}(t)$ and get for $d \geq 3$ :

$$
\begin{aligned}
q^{d+2} \approx\left\|N_{q}\right\|_{2}^{2} & \sim \int_{0}^{\infty} \frac{1}{r} \psi^{2}(r / q) \sum_{a, b \in A_{q}} \frac{J_{\frac{d}{2}-1}(r\|a\|) J_{\frac{d}{2}-1}(r\|b\|)}{(\|a\|\|b\|)^{\frac{d}{2}-1}} d r \\
& \sim \int_{0}^{\infty} r^{d-3} \psi^{2}(r / q) \sum_{a, b \in A_{q}} \hat{\omega}_{B}(r\|a\|) \hat{\omega}_{B}(r\|b\|) d r
\end{aligned}
$$

It is easy to show that in order to get the order of magnitude $q^{d+2}$ for $\left\|N_{q}\right\|_{2}^{2}$ in the latter integral, it suffices to restrict the domain of integration to $(0,1)$. On the other hand, the integral on $[1, \infty)$ would be approximately $q^{-2}\left\|\nu_{q}^{2}\right\|_{2}^{2}$. In the special case $A=\mathbb{Z}^{d}$, the integral (30), taken from 1 to infinity is closely related to the quantity $E_{q}$, see the ensuing Lemma 3.1, However, for a general homogeneous set the integral (30), taken from 1 to infinity apparently cannot be interpreted in terms of the quantity $E_{q}$.

\section{Anisotropic case}

Let us move on to the case of $K$-distances. To proceed, we need the following lemma on the asymptotics of the Fourier transforms $\hat{\omega}_{K}$ and $\hat{\Omega}_{K}$. We do not present a proof here, as for $\hat{\Omega}_{K}$ it can be found in [7], and the case of $\hat{\omega}_{K}$ follows in the same way. For more asymptotics of this kind see [6], [14].

Lemma 2.2 For $\|\xi\| \leq 1, \hat{\omega}_{K}(\xi), \hat{\Omega}_{K}(\xi) \approx 1$, otherwise

$$
\begin{aligned}
& \hat{\omega}_{K}(\xi)=\sum_{j=0}^{1} u_{j}\left(\frac{\xi}{\|\xi\|}\right) J_{\frac{d}{2}-1+j}\left(c_{4}|\xi|_{K *}\right)\|\xi\|^{1-\frac{d}{2}-j}+O\left(\|\xi\|^{-\frac{d+3}{2}}\right), \\
& \hat{\Omega}_{K}(\xi)=\sum_{j=0}^{1} U_{j}\left(\frac{\xi}{\|\xi\|}\right) J_{\frac{d}{2}+j}\left(c_{4}|\xi|_{K *}\right)\|\xi\|^{-\frac{d}{2}-j}+O\left(\|\xi\|^{-\frac{d+5}{2}}\right),
\end{aligned}
$$

where the quantities $u_{0}, U_{0}$ are strictly positive and the constant $c_{4}$ depends on $K$ only.

Without loss of generality, assume $c_{4}=1$ in the formulae (31) above. The sums in the asymptotic expansions have two terms, because this is as many as we will have to analyze. Observe that in the Euclidean case, the expressions (31) reduce to the first term in the sum only.

Lemma 2.2 will be instrumental for our proofs. First let us use it to derive the $K$-analog of the formula (28).

Proposition 2.3 For $d \geq 2$,

$$
\begin{aligned}
\left\|\nu_{q}\right\|_{2}^{2} & \approx \int_{0}^{\infty} r^{d-1} \psi^{2}(r / q) \sum_{a, b \in A_{q}} \hat{\omega}_{K^{*}}(r a) \hat{\omega}_{K^{*}}(r b) d r \\
& \approx \int_{0}^{\infty} r \psi^{2}(r / q) \sum_{a, b \in A_{q}} \frac{J_{\frac{d}{2}-1}\left(|a|_{K} r\right) J_{\frac{d}{2}-1}\left(|b|_{K} r\right)}{\left(|a|_{K}|b|_{K}\right)^{\frac{d}{2}-1}} d r .
\end{aligned}
$$

Proof The proof is direct verification, done by substituting the asymptotic expansion for $\hat{\omega}_{K^{*}}$ (naturally $K^{* *}=K$ ) from (31) into the intermediate term in (32). Given a pair $(a, b) \in A_{q} \times A_{q}$ in the double sum (32), it suffices to consider three cases, as far as the three-term expansions in (31) are concerned: the leading terms for both $a$ and $b$, the leading term for $a$ and the second term for $b$, 
and finally the leading term for $a$ and the remainder for $b$. The contribution of other combinations of terms is negligible, by the order of their asymptotics. Hence the proof has three steps.

1. Given $(a, b)$, take the product of the leading terms in the sum (31). Then, cf. (28), what we get is proportional to the following quantity, involving an integral over $\mathbb{R}^{d}$ :

$$
\begin{gathered}
u_{0}(a /\|a\|) u_{0}(b /\|b\|) \int \hat{\phi}^{2}(\xi / q) \hat{\omega}_{B}\left(|a|_{K} \xi\right) \hat{\omega}_{B}\left(|b|_{K} \xi\right) d \xi \\
\approx \int\left[\phi_{q} *\left(\omega_{B} \circ|a|_{K}^{-1}\right)\right](x) \cdot\left[\phi_{q} *\left(\omega_{B} \circ|b|_{K}^{-1}\right)\right](x) d x
\end{gathered}
$$

where $\omega_{B} \circ|a|_{K}^{-1}(x)=|a|_{K}^{1-d} \omega_{B}\left(|a|_{K}^{-1} x\right)$. The integrand in the right hand side of (33) is roughly the product of characteristic functions of concentric Euclidean annuli, of radii $|a|_{K}$ and $|b|_{K}$. It vanishes if $\left.|| a\right|_{K}-|b|_{K} \mid>\frac{1}{q}$, and its integral is proportional to $|a|_{K}^{d-1}$ in case $|a|_{K}=|b|_{K}$. Thus the average value of $\omega_{B} \circ|a|_{K}^{-1}$ across the Euclidean annulus of radius $|a|_{K}$ and width of $\approx \frac{1}{q}$ is proportional to $q$. So we get

$$
\begin{aligned}
\sum_{a, b \in A_{q}} u_{0}(a /\|a\|) u_{0}(b /\|b\|) \int_{0}^{\infty} r \psi^{2}(r / q) \frac{J_{\frac{d}{2}-1}\left(|a|_{K} r\right) J_{\frac{d}{2}-1}\left(|b|_{K} r\right)}{\left(|a|_{K}|b|_{K}\right)^{\frac{d}{2}-1}} d r & \approx q \sum_{k=1}^{\sim q^{2}} \frac{\Gamma^{2}\left(r_{k}, \delta\right)}{r_{k}^{d-1}} \\
& \approx \int_{0}^{\infty} \nu_{q}^{2}(t) d t .
\end{aligned}
$$

The sum in the intermediate expression is taken over consecutive $K$-annuli of radius $r_{k}$ and fixed width $\delta \sim \frac{1}{q}$, cf. (17).

2. Now let us take the first term in the sum (31) for $a$ and the second one for $b$ and substitute them in the right-hand side of (32). Note that merely using the leading order asymptotics in this case would result in a superfluous factor $\int_{1}^{\infty} r^{-1} \psi^{2}(r / q) d r \approx \log q$.

So, given $(a, b) \in A_{q} \times A_{q}$ we have, similarly to (33) and omitting uniform constants:

$$
\begin{aligned}
\int_{0}^{\infty} \psi^{2}(r / q) \frac{J_{\frac{d}{2}-1}\left(|a|_{K} r\right) J_{\frac{d}{2}}\left(|b|_{K} r\right)}{|a|_{K}^{\frac{d}{2}-1}|b|_{K}^{\frac{d}{2}}} d r & \sim \int \hat{\phi}^{2}(\xi / q) \hat{\omega}_{B}\left(|a|_{K} \xi\right) \hat{\Omega}_{B}\left(|b|_{K} \xi\right) d \xi \\
& =\int\left[\phi_{q} *\left(\omega_{B} \circ|a|_{K}^{-1}\right)\right](x) \cdot\left[\phi_{q} *\left(\Omega_{B} \circ|b|_{K}^{-1}\right)\right](x) d x \\
& \approx \begin{cases}\frac{1}{|a|_{K}^{d-1}|b|_{K}^{d}}|a|_{K}^{d-1}, & |a|_{K} \leq|b|_{K}+\frac{1}{q}, \\
0 & \text { otherwise. }\end{cases}
\end{aligned}
$$

Summing in absolute value over $a, b \in A_{q}$ yields

$$
\sum_{b \in A_{q}}|b|_{K}^{-d} \sum_{|a|_{K} \leq|b|_{K}} 1 \approx q^{d}
$$

cf. (23).

3. Finally, we estimate the contribution into (32) of the leading order term for $a$ and the remainder for $b$ in (31). Rewrite the integral in (32) as $\sum_{a, b \in A_{q}} I_{a, b}$ and notice that without loss of generality one can assume $|a|_{K} \geq|b|_{K}$. Then partition

$$
\sum_{a, b \in A_{q},|b|_{K} \leq|a|_{K}} I_{a, b}=\sum_{a, b \in A_{q},|b|_{K} \leq|a|_{K}}\left(\int_{0}^{|a|_{K}^{-1}}+\int_{|a|_{K}^{-1}}^{|b|_{K}^{-1}}+\int_{|b|_{K}^{-1}}^{\infty}\right) .
$$


In the first piece we substitute 1 for the $a$-term and 1 for the $b$-term, this yields

$$
\sum_{a \in A_{q}} \sum_{b \in A_{q},|b|_{K} \leq|a|_{K}} \int_{0}^{|a|_{K}^{-1}} r^{d-1} d r \approx \sum_{a \in A_{q}}|a|_{K}^{-d} \sum_{b \in A_{q},|b|_{K} \leq|a|_{K}} 1 \approx \sum_{a \in A_{q}} 1 \approx q^{d} .
$$

For the second piece use 1 for the $b$-term and zero order asymptotics $\left(|a|_{K} r\right)^{-\frac{d-1}{2}}$ for the $a$-term, this yields

$$
\sum_{a \in A_{q}} \sum_{b \in A_{q},|b|_{K} \leq|a|_{K}} \int_{|a|_{K}^{-1}}^{|b|_{K}^{-1}}|a|_{K}^{\frac{1-d}{2}} r^{\frac{d-1}{2}} d t \approx \sum_{a \in A_{q}}|a|_{K}^{\frac{1-d}{2}} \sum_{b \in A_{q},|b|_{K} \leq|a|_{K}}|b|_{K}^{-\frac{d+1}{2}} \approx \sum_{a \in A_{q}} 1 \approx q^{d}
$$

Finally, for the third piece substitute $\left(|a|_{K} r\right)^{-\frac{d-1}{2}}$ for the $a$-term terms and $\left(|b|_{K} r\right)^{-\frac{d+3}{2}}$, for the $b$-term to get

$$
\left(\sum_{a, b \in A_{q}}|a|_{K}^{-\frac{d-1}{2}}|b|_{K}^{-\frac{d+3}{2}}\right) \cdot \int_{1}^{\infty} \psi^{2}(r / q) t^{-2} d t \lesssim q^{d-1} .
$$

We have shown that the upper estimates in steps $\mathbf{2 , 3}$ match the lower bound (23) for $\left\|\nu_{q}\right\|_{2}^{2}$. This completes the proof of Proposition $2.3 \square$.

\section{Poisson formula}

From now on consider the case $A=\mathbb{Z}^{d}, A_{q}=\mathbb{Z}_{q}^{d}$. Then the quantities $\nu_{q}(t), N_{q}(t), E_{q}(t)$ can be computed directly, rather than via (24), using the Poisson summation formula. By doing this, one gets expressions for these quantities on the $t$-side, rather than on the Hankel transform side, cf. (29). For example, see (13), (15), for $\frac{1}{q}<t<q-\frac{1}{q}$ one has

$$
\nu_{q}(t)=t^{\frac{1-d}{2}} \sum_{a \in \mathbb{Z}^{d}} \int \omega_{K}(x / t) \phi_{q}(x-a) d x
$$

Applying the Poisson summation formula to the convolution that the integral in (41) represents and doing the same thing for the quantities $N_{q}, E_{q}$ yields, for $\frac{1}{q}<t<q-\frac{1}{q}$ :

$$
\begin{aligned}
& \nu_{q}(t) \sim t^{\frac{d-1}{2}} \sum_{a \in \mathbb{Z}^{q}} \hat{\phi}(a / q) \hat{\omega}_{K}(t a) \equiv \nu(t), \\
& N_{q}(t) \sim t^{\frac{d+1}{2}} \sum_{a \in \mathbb{Z}^{q}} \hat{\phi}(a / q) \hat{\Omega}_{K}(t a) \equiv N(t), \\
& E_{q}(t) \sim t^{\frac{d+1}{2}} \sum_{a \in \mathbb{Z}^{q} \backslash\{0\}} \hat{\phi}(a / q) \hat{\Omega}_{K}(t a) \equiv E(t) .
\end{aligned}
$$

Note however that the quantities in the right hand side of (42) are unbounded as $t \rightarrow \infty$, besides the summation is carried over the whole integer lattice. Still, there is a considerable resemblance between the expression for $\nu(t)$ and the square root of the integrand in (32). Let us introduce $L^{2}\left(\mathbb{R}_{+}\right)$quantities

$$
[\tilde{\nu}(t), \tilde{N}(t), \tilde{E}(t)]=\psi(t / q)[\nu(t), N(t), E(t)]
$$

Clearly

$$
\left\|\nu_{q}\right\|_{2}^{2} \lesssim\|\tilde{\nu}\|_{2}^{2}, \quad \int_{0}^{q} E_{q}^{2}(t) d t \lesssim\|\tilde{E}\|_{2}^{2}
$$


Now define the quantities $\nu_{q *}(t), \nu_{*}(t), \tilde{\nu}_{*}(t)$ with respect to the dual body $K^{*}$ in the same way as $\nu_{q}(t), \nu(t), \tilde{\nu}(t)$, have been defined for $K$ (see (13), (15), (43), (42)), do the same thing for the quantity $E$. Also with respect to $K^{*}$, define the notation $\Gamma_{*}$, cf. (17). Note that $\tilde{\nu}_{*}(t)$ will be given precisely by the square root of the integrand in (32), after the summation therein has been extended over the whole $\mathbb{Z}^{d}$ by weighing each term with $\hat{\phi}(a / q)$. Since the dual quantities also satisfy (44), comparison of the expression for $\nu$ in (42) with (32) results in the following theorem.

Theorem 3 For $d \geq 2$,

$$
\|\tilde{\nu}\|_{2} \approx\left\|\tilde{\nu}_{*}\right\|_{2}
$$

Note that in the definition (43) of the quantity $\tilde{\nu}$ there is no harm restricting the summation to $\mathbb{Z}^{d} \backslash\{0\}$, which will be done further. Indeed, $a=0$ results in a regular term $t^{\frac{d-1}{2}}$, and it is easy to check that the contribution of this term into $\|\tilde{\nu}\|_{2}^{2}$ is $O\left(q^{d}\right)$, cf. (23).

The next lemma is the central ingredient to prove Theorem 2.

Lemma 3.1 For $d \geq 2$ :

$$
\|\tilde{E}\|_{2}^{2} \lesssim \int_{0}^{\infty} \frac{\tilde{\nu}_{*}^{2}(t)}{1+t^{2}} d t+R(q)
$$

where $R(q)=O\left(q^{d-2}\right)$ in $d \geq 3$ and $O(\log q)$ in $d=2$.

Proof The proof follows the same pattern as the proof of Proposition 2.3. Namely, it consists in direct verification, done by substituting the asymptotic expansion for $\hat{\Omega}_{K}$ from (31) into the definition (42), (43) of $\tilde{E}$ and evaluating the second moment. Given $a, b \in \mathbb{Z}^{d} \backslash\{0\}$ in the resulting double sum, it suffices to consider three cases: the leading terms for both $a$ and $b$, the leading term for $a$ and the second term for $b$, and finally the leading term for $a$ and the remainder for $b$. Hence the proof has three steps.

1. For the principal terms' contribution into $\|\tilde{E}\|_{2}^{2}$, omitting uniform positive constants we get

$$
\sum_{a, b \in \mathbb{Z}^{d} \backslash\{0\}} \hat{\phi}(a / q) \hat{\phi}(b / q) \int_{0}^{\infty} t \psi^{2}(t / q) \frac{J_{\frac{d}{2}}\left(|a|_{K *} t\right) J_{\frac{d}{2}}\left(|b|_{K *} t\right)}{\left(|a|_{K *} b_{K *}\right)^{\frac{d}{2}}} d t .
$$

The integral in (47), given $(a, b)$ can be rewritten as an integral over $\mathbb{R}^{d}$ :

$$
\begin{array}{r}
\int\left[( \Omega _ { B } \circ | a | _ { K * } ^ { - 1 } ) * \phi _ { q } \left\lceil( \xi ) \xi \cdot \left[\left(\Omega_{B} \circ|b|_{K *}^{-1}\right) * \phi_{q}\lceil(\xi) \xi d \xi\right.\right.\right. \\
=\int \nabla_{x}\left[\left(\Omega_{B} \circ|a|_{K *}^{-1}\right) * \phi_{q}\right](x) \cdot \nabla_{x}\left[\left(\Omega_{B} \circ|b|_{K *}^{-1}\right) * \phi_{q}\right](x) d x .
\end{array}
$$

where, cf. (33), $\Omega_{B} \circ|a|_{K *}^{-1}(x)=|a|_{K *}^{-d} \Omega_{B}\left(|a|_{K *}^{-1} x\right)$. The integral in the right-hand side of (48) is clearly zero if $\left.|| a\right|_{K^{*}}-|b|_{K^{*}} \mid>\frac{1}{q}$, while if $|a|_{K^{*}}=|b|_{K^{*}}$, it is $O\left(|a|_{K^{*}}^{d-1}\right)$. Hence, (47) is

$$
\approx q \sum_{k=1}^{\infty} \frac{\Gamma_{*}^{2}\left(r_{k}, \delta\right)}{r_{k}^{2} r_{k}^{d-1}} \psi^{2}\left(r_{k} / q\right) \approx \int_{0}^{\infty} \frac{\tilde{\nu}_{*}^{2}(t)}{1+t^{2}} d t
$$

cf. (34). 
2. For the principal $a$-term and second $b$-term in the asymptotics (31), omitting uniform constants, we get

$$
\sum_{a, b \in \mathbb{Z}^{d} \backslash\{0\}} \hat{\phi}(a / q) \hat{\phi}(b / q) \int_{0}^{\infty} \psi^{2}(t / q) \frac{J_{\frac{d}{2}}\left(|a|_{K *} t\right) J_{\frac{d}{2}+1}\left(|b|_{K *} t\right)}{|a|_{K *}^{\frac{d}{2}} b_{K *}^{\frac{d}{2}+1}} d t .
$$

Observe that the expression (50) is reminiscent of (35), only in dimension $d+1$. Let $w_{B}, W_{B}$ be the Lebesgue measure on $S^{d}$ and the characteristic function of the Euclidean unit ball in $\mathbb{R}^{d+1}$, respectively. Let $(y, \zeta) \in \mathbb{R}^{d+1} \times \mathbb{R}^{d+1}$, let the radial cutoff function $\varphi$ be defined in the same way as $\phi$, only in dimension $d+1$.

Denote $\varphi_{q}(y)=q^{d+1} \varphi(q y), w_{B} \circ|a|_{K *}^{-1}(y)=|a|_{K *}^{-d} w_{B}\left(|a|_{K *}^{-1} y\right)$, as well as $W_{B} \circ|a|_{K *}^{-1}(y)=$ $|a|_{K *}^{-d-1} W_{B}\left(|a|_{K *}^{-1} y\right)$. Then given $(a, b)$, the integral in (50) is a constant times

$$
\begin{aligned}
& \int\left[( w _ { B } \circ | a | _ { K * } ^ { - 1 } ) * \varphi _ { q } \left\lceil( \zeta ) \cdot \left(\left[\left(W_{B} \circ|a|_{K *}^{-1}\right) * \varphi_{q}\lceil(\zeta)\|\zeta\|) d \zeta\right.\right.\right.\right. \\
& \approx \int\left[\left(w_{B} \circ|a|_{K *}^{-1}\right) * \varphi_{q}\right](y) \cdot\left\|\nabla_{y}\left[\left(W_{B} \circ|a|_{K *}^{-1}\right) * \varphi_{q}\right](y)\right\| d y .
\end{aligned}
$$

Thus the integral vanishes if $\left.|| a\right|_{K^{*}}-|b|_{K^{*}} \mid>\frac{1}{q}$ and is approximately $\frac{1}{|b|_{K^{*}}^{d+1}}$ if $|a|_{K^{*}}=|b|_{K^{*}}$. Summation in absolute values over $(a, b)$ values results precisely in (49).

3. We deal with the remainder in the asymptotics (31) in the same way as it was done in Proposition 2.3. On this step, in the double sum in $a, b \in \mathbb{Z}^{d} \backslash\{0\}$ representing the second moment of $\tilde{E}$ we use the leading term for $a$ and the remainder for $b$. The demonstration consists in essentially repeating (37-40). The presence of the cutoff terms $\hat{\phi}(a / q), \hat{\phi}(b / q)$ allows here for restricting the summation to $\mathbb{Z}_{q}^{d} \backslash\{0\}$. Assume $|a|_{K^{*}} \geq|b|_{K^{*}}$ and partition each integral into three counterparts in the summation according to (37).

For instance, for the first counterpart, cf. (38), we have

$$
\begin{aligned}
\sum_{a \in \mathbb{Z}_{q}^{d} \backslash\{0\}} \sum_{b \neq 0,|b|_{K^{*}} \leq|a|_{K^{*}}} \int_{0}^{|a|_{K^{*}}^{-1}} r^{d+1} d r & \approx \sum_{a \in \mathbb{Z}_{q}^{d} \backslash\{0\}}|a|_{K^{*}}^{-2} \\
& \approx\left\{\begin{array}{cc}
q^{d-2}, & d \geq 3, \\
\log q, & d=2 .
\end{array}\right.
\end{aligned}
$$

The estimates of the second and third counterpart are done along the same lines as (39) and (40) and we omit them.

Remark It is clear that estimating the right-hand-side of (46) which is essentially an $L^{2}$-estimate for $\frac{\nu}{t}$ does not suffice to get a sharp estimate for $\nu$, when it grows in average slower than $\sqrt{t}$ as $t \rightarrow \infty$. That is why we cannot prove Theorem 2 for $d=2$. The logarithmic factor in the case $d=3$ in the estimate (21) also appears to be an artifact.

\section{Proof of Theorem 2}

Theorem 2 will follow immediately from the bound (46) of Lemma 3.1 and the following lemma, which somewhat generalizes the results of [8].

Lemma 4.2 We have the following bound:

$$
\|\tilde{E}\|_{2}^{2} \lesssim b_{d}(q), \text { where } b_{d}(q)= \begin{cases}q^{d-2}, & d \geq 4 \\ q \log ^{2} q, & d=3 \\ q, & d=2\end{cases}
$$


Proof There is no harm changing in(46) the lower limit of integration to 1 and $1+t^{2}$ in the denominator to $t^{2}$.

By definition of $\tilde{\nu}$, for any $t_{l}, t_{u}$, with $t_{u}-t_{l} \gg \frac{1}{q}$ and a small enough $\delta \sim \frac{1}{q}$, we have the representation of the integral as a Darboux sum:

$$
\int_{t_{l}}^{t_{u}} \frac{\tilde{\nu}_{*}^{2}(t)}{t^{2}} d t \approx \sum_{k} \frac{\left.\nu_{*}^{2}(t)\right|_{t \in I_{k}}}{t_{k}^{2}} \psi\left(t_{k} / q\right) \delta
$$

where the intervals $I_{k}=\left[t_{k}, t_{k+1}\right)$ of length $\delta$ partition $\left[t_{l}, t_{u}\right)$ and choice of $t \in\left[t_{k}, t_{k+1}\right)$ is arbitrary.

Then one can always choose $t$ inside each interval $I_{k}$ in such a way that

$$
\nu_{*}(t) \lesssim \max \left[t^{\frac{d-1}{2}}, q\left|E_{*}\right|(t)\right]
$$

Indeed, the first term inside the above maximum corresponds to the case of the existence of $t \in I_{k}$ such that $\nu_{*}(t) \lesssim t^{\frac{d-1}{2}}$. Otherwise, let us use the fact that $\frac{d E_{*}(t)}{d t} \approx \nu_{*}(t)+O\left(t^{\frac{d-1}{2}}\right)$, and if $\nu_{*}(t) \gtrsim t^{\frac{d-1}{2}}$, the $O\left(t^{\frac{d-1}{2}}\right)$ term can be omitted. Then $\left|E_{*}(t)\right| \gtrsim \int_{t_{0}}^{t} \nu_{*}(\tau) d \tau$, where at $t_{0},\left|E_{*}\right|$ has its absolute minimum in $I_{k}$. Which implies that $q \sup _{I_{k}}\left|E_{*}(t)\right| \gtrsim \inf _{I_{k}} \nu_{*}(t)$ in this case.

Note that due to (23) all the "regular" terms $O\left(t^{\frac{d-1}{2}}\right)$ that appear further would a-priori result in (53), and in fact stronger inequalities for $d=2,3$.

Furthermore by (55)

$$
\int_{1}^{\infty} \frac{\tilde{\nu}_{*}^{2}(t)}{t^{2}} d t \lesssim \int_{1}^{\infty} t^{d-3} \psi(t / q) d t+\int_{\mathcal{J}} \frac{\tilde{\nu}_{*}^{2}(t)}{t^{2}} d t
$$

where

$$
\mathcal{J}=\left\{t: \nu_{*}(t) \leq c_{5} q\left|E_{*}(t)\right|\right\},
$$

for some $c_{5}$. The first integral in (56) bounded via $q^{d-2}$ for $d \geq 3$ and $\log q$ for $d=2$.

Let us turn to the second integral in (56). Clearly, in order to get the upper bound, the integral can be extended from $\mathcal{J}$ to $\mathbb{R}_{+}$, under the assumption that $\nu_{*}(t) \leq c_{5} q\left|E_{*}(t)\right|$ everywhere (note that $\mathcal{J}$ can be represented as the union of intervals of length not smaller than $\approx \frac{1}{q}$ each). Under this assumption, we write out a dyadic decomposition:

$$
\int_{1}^{\infty} \frac{\tilde{\nu}_{*}^{2}(t)}{1+t^{2}} d t \approx \sum_{k=0}^{\infty} 2^{-2 k} \int_{2^{k}}^{2^{k+1}} \tilde{\nu}_{*}^{2}(t) d t \lesssim q \sum_{k=0}^{\infty}\left|\psi\left(2^{k} / q\right)\right| 2^{\frac{d+1}{4} k-2 k} \sqrt{\int_{2^{k}}^{2^{k+1}}\left|\tilde{E}_{*}\right|^{2} \nu_{*}(t) d t},
$$

To get the right-hand side we have applied Cauchy-Schwartz and used the fact that in an annulus of width $2^{k}$ the integral of $\nu_{*}$ is $O\left(2^{k \frac{d+1}{2}}\right)$, recall the scaling (15).

Furthermore, using the fact that $\frac{d E(t)}{d t} \approx \nu_{*}(t)+O\left(t^{\frac{d-1}{2}}\right)$, we have

$$
\int_{2^{k}}^{2^{k+1}}\left|\tilde{E}_{*}\right|^{2} \nu_{*}(t) d t \lesssim\left|\psi\left(2^{k} / q\right)\right|\left(\left|E_{*}\left(2^{k}\right)\right|^{3}+\left|E_{*}\left(2^{k+1}\right)\right|^{3}+2^{k \frac{d-1}{2}} \int_{2^{k}}^{2^{k+1}} E_{*}^{2}(t) d t\right) .
$$

The cubic terms in brackets are bounded as

$$
O\left[2^{3 k\left(\frac{d-3}{2}+\frac{2}{d+1}\right)}+2^{3 k \frac{d-1}{2}} q^{-3}\right]
$$


which follows from the well known, see e.g. [10], $L^{\infty}$ estimate

$$
\left|E_{0}(t)\right| \lesssim t^{d-2+\frac{2}{d+1}}+q^{-1} t^{d-1}
$$

where $E_{0}(t)=t^{\frac{d-1}{2}} E(t)$, in view of the scaling (15). It is a routine calculation to show using the decay of $\psi$ that the contribution of these terms into (58) is well in compliance with (53).

Hence we are left with

$$
\int_{0}^{\infty} \tilde{E}^{2}(t) d t \lesssim b_{d}(q)+q \sum_{k=0}^{\infty} 2^{k\left(\frac{d}{2}-2\right)}\left|\psi\left(2^{k} / q\right)\right| \sqrt{\int_{2^{k}}^{2^{k+1}} \tilde{E}_{*}^{2}(t) d t} .
$$

Assuming that the sum above is $\gtrsim b_{d}(q)$, see (53), consider the case $d \geq 4$ first. Then, as clearly

$$
\sum_{k=0}^{\infty} 2^{k\left(\frac{d}{2}-2\right)}\left|\psi\left(2^{k} / q\right)\right| \lesssim q^{\frac{d}{2}-2}, \text { for } d \geq 4,
$$

we have

$$
\int_{0}^{\infty} \tilde{E}^{2}(t) d t \lesssim q^{\frac{d}{2}-1} \sqrt{\int_{0}^{\infty} \tilde{E}_{*}^{2}(t) d t}
$$

and it follows that $\|\tilde{E}\|_{2}^{2},\left\|\tilde{E}_{*}\right\|_{2}^{2} \lesssim b_{d}(q)=q^{d-2}, d \geq 4$, as one can certainly swap the subscript * to the left-hand side.

The case $d=2,3$ requires some extra consideration, see [8], which we have adopted from the latter reference for the sake of completeness. Recall that the quantity $E$ has been defined with respect to the parameter $q$, where $\frac{1}{q}$ is the characteristic scale of the smoothing. To reflect this fact, let us further write $E=E^{(q)}, \tilde{E}=\tilde{E}^{(q)}$. It is easy to verify by definition of $E$ that for $t \lesssim \bar{q} \lesssim q$, one has

$$
\left|E^{(\bar{q})}\right|(t) \lesssim\left|E^{(q)}\right|(t)+O\left(t^{\frac{d-1}{2}} \bar{q}^{-1}\right) .
$$

Let us rewrite (62) as follows:

$$
\int_{0}^{\infty}\left|\tilde{E}^{(q)}\right|^{2}(t) d t \lesssim b_{d}(q)+q \sup _{k}\left(\sqrt{\frac{\int_{0}^{2^{k+1}}\left|\tilde{E}_{*}^{(q)}\right|^{2}(t) d t}{b_{d}\left(2^{k+1}\right)}}\right) \sum_{k=0}^{\infty} 2^{k\left(\frac{d}{2}-2\right)}\left|\psi\left(2^{k} / q\right)\right| \sqrt{b_{d}\left(2^{k+1}\right)} .
$$

Evaluating the sum yields

$$
\frac{\int_{0}^{\infty}\left|\tilde{E}^{(q)}\right|^{2} d t}{b_{d}(q)} \lesssim 1+\sup _{k}\left(\sqrt{\frac{\int_{0}^{2^{k+1}}\left|\tilde{E}_{*}^{(q)}\right|^{2} d t}{b_{d}\left(2^{k+1}\right)}}\right) .
$$

The supremum above should be achieved for some finite $k$, because of the decay, built into the quantity $\tilde{E}$, due to the presence of the cutoff $\psi$. Then define $\bar{k}$ as follows:

$$
m(q)=\max \sup _{k}\left(\frac{\int_{0}^{2^{k+1}}\left|\tilde{E}^{(q)}\right|^{2} d t}{b_{d}\left(2^{k+1}\right)}, \frac{\int_{0}^{2^{k+1}}\left|\tilde{E}_{*}^{(q)}\right|^{2} d t}{b_{d}\left(2^{k+1}\right)}\right)
$$

is achieved for $k=\bar{k}$. Without loss of generality suppose the maximum in (68) is effected by the first entry. Also suppose $m(q)>1$, otherwise the proof of Lemma 4.2 would be complete. 
Then by definition of $\bar{k}$,

$$
\frac{\int_{0}^{\infty}\left|\tilde{E}^{(q)}\right|^{2} d t}{b_{d}(q)} \lesssim \frac{\int_{0}^{\infty}\left|\tilde{E}^{(q)}\right|^{2} d t}{b_{d}\left(2^{\bar{k}+1}\right)},
$$

hence $2^{\bar{k}+1} \lesssim q$. Consider now two cases, with the objective to show that $m(q)=O(1)$.

Case 1. If $2^{\bar{k}+1} \gtrsim q$, then the quantity $m(q)$, by its definition, has to be bounded by a constant times the left-hand side of (67). This implies $m(q)=O(1)$.

Case 2. Suppose now $2^{\bar{k}+1} \ll q$, let $\bar{q}=2^{\bar{k}+1}$. Look back at the expressions (67) and (68) replacing $q$ by $\bar{q}$, i.e. as the statements about the quantity $\tilde{E}^{(\bar{q})}$ rather than $\tilde{E}^{(q)}$. By $(65)$, if $k=\bar{k}$, then

$$
\int_{0}^{2^{k+1}}\left|\tilde{E}^{(\bar{q})}\right|^{2} d t \approx \int_{0}^{2^{k+1}}\left|\tilde{E}^{(q)}\right|^{2} d t
$$

Otherwise, if $k<\bar{k}$, the relation (70) should in general hold with the $\lesssim$ sign.

This implies $m(\bar{q}) \approx m(q)$, in other words $m(\bar{q})$ may be thought to be achieved when $k=\bar{k}$, so by $(70)$

$$
m(\bar{q}) \lesssim \frac{\int_{0}^{\infty}\left|\tilde{E}^{(\bar{q})}\right|^{2} d t}{b_{d}(\bar{q})}
$$

This, similarly to Case 1 , the statement (67) for the quantity $\tilde{E}^{(\bar{q})}$, would imply $m(\bar{q})=O(1)$, so once again $m(q)=O(1)$.

Therefore the right-hand side of (67) always turns our to be $O(1)$. This completes the proof of Lemma 4.2 and Theorem 2.

Acknowledgements: Research has been partially supported by the NSF Grant DMS02-45369, EPSRC Grant GR/S13682/01 and the Bristol Institute for Advanced Study.

\section{References}

[1] G. Arutyunyants, A. Iosevich. Falconer conjecture, spherical averages, and discrete analogs. Contemp. Math. 342 "Towards the theory of geometric graphs", J. Pach editor (2004) 15-25.

[2] J. Bourgain. Hausdorff dimension and distance sets. Israel Math. J. 87 (1994) 193-201.

[3] B. Erdogan. On the Falconer conjecture. Preprint 2004.

[4] K. J. Falconer. The geometry of fractal sets. Cambridge Univ. Press 1985.

[5] K. J. Falconer. On the Hausdorff dimensions of distance sets. Mathematika 32 (1986) 206-212.

[6] C. S. Herz. Fourier transforms related to convex sets. Ann. Math. 75 (1962) no 1, 81-92.

[7] A. Iosevich, M. Rudnev. A combinatorial approach to orthogonal exponentials. IMRN $\mathbf{5 0}$ (2003), 2671-2685.

[8] A. Iosevich, E. Sawyer, A. Seeger. Mean square discrepancy bounds for the number of lattice points in large convex bodies. J. D'Anal. Maths. 87 (2002), 209-230.

[9] A. Iosevich, E. Sawyer, A. Seeger. Mean square discrepancy bounds for the number of lattice points in large convex bodies II: planar domains. (preprint) (2004). 
[10] E. Landau. Vorlesungen über Zahlentheorie, Vol. II. S. Hirzel, Leipzig 1927.

[11] P. Mattila. Spherical averages of Fourier transforms of measures with finite energy; dimension of intersection and distance sets. Mathematica 34 (1987), 207-228.

[12] W. Müller. On the average order of the lattice rest of a convex body. Acta Arith. 80 (1997) 89-100.

[13] J. Pach, M. Sharir. Geometric Incidences. Towards a theory of geometric graphs, Contemp. Math., 342, Amer. Math. Soc., Providence, RI, 2004.

[14] C. Sogge. Fourier integrals in classical analysis. Cambridge University Press 1993.

[15] G.N. Watson. A Treatise on the Theory of Bessel Functions, 2nd ed. Cambridge University Press 1966.

[16] T. Wolff. Decay of circular means of Fourier transforms of measures. IMRN 10 (1999), 547-567.

[17] T. Wolff. Lectures in harmonic analysis. Caltech Class Lecture Notes, revised by I. Łaba, 2002.

Alex Iosevich: University of Missouri, Columbia, Missouri, 65211 USA, iosevich@math.missouri.edu Mischa Rudnev: University of Bristol, Bristol BS6 6AL UK, m.rudnev@bris.ac.uk 